\title{
A simple chemical method for the preparation of silver nanocables from self-assembling tubular $\mathrm{Ag}^{+}$precursors
}

\author{
Oliver Weichold, ${ }^{* a}$ Shou-Chian Hsu ${ }^{b}$ and Martin Möller ${ }^{a}$
}

\author{
Received 1st September 2006, Accepted 3rd October 2006 \\ First published as an Advance Article on the web 17th October 2006 \\ DOI: $10.1039 / \mathrm{b} 612671 \mathrm{e}$
}

\begin{abstract}
A simple and efficient method for the in situ fabrication at ambient temperature of nanocables consisting of a silver core and an insulating organic shell from a self-assembling silver precursor is presented. The precursor assembles into tubular aggregates several microns in length, which template the formation of silver wires, under defined reaction conditions. Proper control of the reaction conditions, such as reagent concentrations and the method of reduction, is important in order to obtain well-defined nanocables as individual dispersed entities. These consist of 30-45 nm silver cores, 60-70 nm walls, and an aspect ratio of $>200$. IR spectroscopy revealed the organic sheath to be cross-linked via a bridging-type coordination to the silver ions, which helps in the formation of the tubular aggregation and provides structural rigidity.
\end{abstract}

\section{Introduction}

Due to their special chemical and physical properties, nanostructured materials currently rank among the most challenging and highly pursued fields in both basic and applied research and are believed to form the basis of future high-tech industries. Particularly one-dimensional (1D) nanostructured materials, such as nanorods, nanotubes, and nanowires, have attracted much research interest in recent years due to their unique quantum properties and potential applications in many fields. For instance, bifunctional gold/nickel nanorods are potentially attractive carriers for the delivery of DNA into cells by the control of the nanorods' size and composition. ${ }^{1}$ Gold nanorods have been used as contrast agents for biological imaging to detect diseases at an early stage. ${ }^{2}$ A highly efficient diode made by using an electric field to create semiconducting $\mathrm{p}$ and $\mathrm{n}$ regions on a single-wall carbon nanotube has been reported. ${ }^{3}$ Functioning high-frequency circuits from nanoscale building blocks have recently been assembled successfully. Based on silicon nanowires, these devices can be applied in lightweight, portable electronics. ${ }^{4}$

Nanowires with sheaths, which are known as nanocables, are a new kind of practical 1D nanostructure. Their functions can be further improved through a rational design of wire and sheath from different materials. For example, chemically inert materials such as silica around metallic or semiconductor nanowires can provide electric insulation and prevent surface oxidation. $^{5-10}$ Polymer sheaths, such as poly(vinyl acetate) ${ }^{11}$ (PVAc) and poly(vinyl alcohol), ${ }^{12}$ have been used to increase the dispersability of metal or semiconductor cores in organic solvents. In addition, a fully organic inverse cable was made by wrapping polypyrrole around poly(methyl methacrylate) (PMMA). ${ }^{13}$ Here, the PMMA is used to increase the

${ }^{a} D W I$ an der RWTH Aachen e. V., Pauwelsstrasse 8, 52056 Aachen, Germany.E-mail: weichold@dwi.rwth-aachen.de; Fax: +492418023301; Tel: +492418023300

${ }^{b}$ Institute of Materials Science Engineering, Chiao Tung University,

1001 Ta Hsueh Road, Hsin Chu, Taiwan 300, Republic of China mechanical properties of the conducting polymer-composite and to make it easier to process.

Recently, different methods such as laser ablation combined with thermal evaporation, ${ }^{5-8}$ sol-gel reactions, ${ }^{14}$ templating, ${ }^{10,11,13-17}$ hydrothermal conversion, ${ }^{12,18}$ and capillary force ${ }^{19,20}$ have been employed for the preparation of nanocables. Using structure-directing templates is a highly efficient method, since the cable structure is readily formed. For example, $\mathrm{Ni}-\mathrm{Cu},{ }^{16} \mathrm{Co}-\mathrm{Ge},{ }^{14}$ and $\mathrm{Ni}-\mathrm{V}_{2} \mathrm{O}_{5}{ }^{17}$ nanocables have been prepared in the pores of commercial membranes by electrochemical deposition. Likewise, Ag nanocables have been made by thermal decomposition of $\mathrm{AgNO}_{3}$ absorbed in mesoporous silica. ${ }^{10}$ When using self-assembling templates, the desired core-shell structure becomes thermodynamically favourable and forms by itself. In this way, CdSe-PVAc nanocables have been prepared under $\gamma$-irradiation. ${ }^{11}$

In this paper, we report a simple yet versatile wet-chemical approach for the one-pot preparation of individual $\mathrm{Ag}$ nanocables using the self-assembly of in situ prepared diamminesilver-(I) oleate.

\section{Experimental}

\section{Materials and methods}

Oleic acid (90\% purity) was purchased from Aldrich. Formaldehyde solution ( $37 \%$ in water) and silver nitrate were obtained from Merck and were of analytical grade. Reagent grade ammonium hydroxide solution (25\% in water) was purchased from Riedel-de Haën. Reagent grade ethanol and potassium hydroxide were purchased from KMF (Siegburg, Germany). All materials were used as received without further purification. Water was purified through a Pure Lab plus UV system (USF EIGA). All the reactions in this study were carried out at ambient conditions.

IR spectra were recorded on a Nicolet Thermo Nexus FTIR spectrometer. The optical microscopy textures were obtained using a Zeiss Axioplan 2 with calibrated image-processing software. The products were visualized at room temperature 

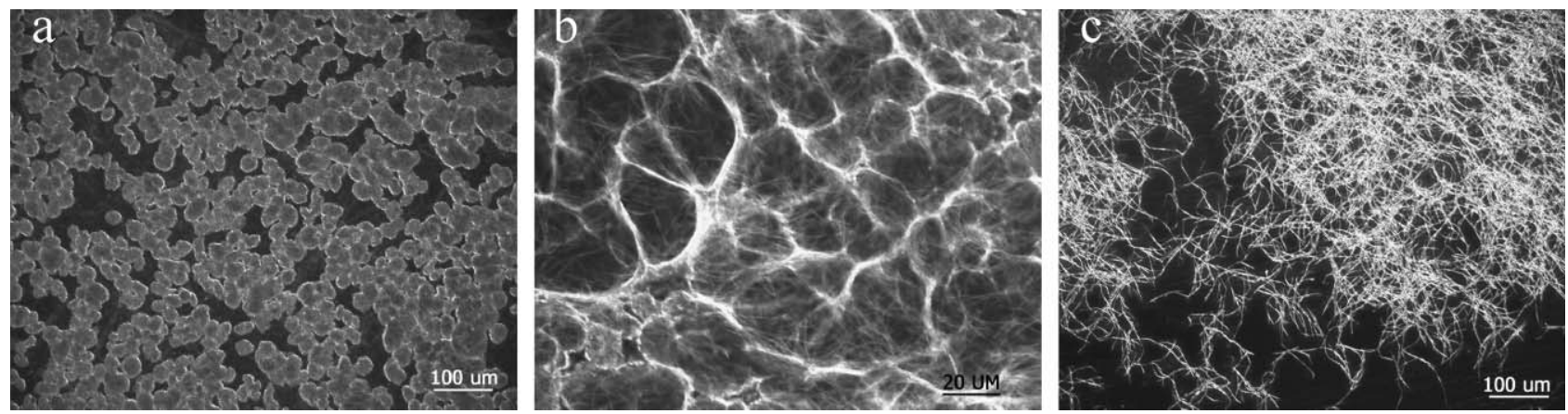

Fig. 1 Dark-field optical micrographs of a diamminesilver-(I) oleate gel at an OA/Ag molar ratio of 30 before (a, b) and after (c) dispersion in ethanol.

between a glass slide and cover slip. Bright-field transmission electron microscopy (TEM) images were recorded using a Philip CM 10 transmission electron microscope. The dark-field TEM image and the elemental profile across a nanocable were obtained on a FEI TECNAI F20. The samples before reduction were prepared by dispersing the viscous white gels in ethanol. The reduced samples were first centrifuged at $4000 \mathrm{rpm}$ for $10 \mathrm{~min}$ and then redispersed in ethanol. Carboncoated copper grids were used as support.

\section{Preparation}

In a typical procedure, the diamminesilver-(I) complex (1), was prepared by the dropwise addition of an ammonium hydroxide solution into $1.8 \mathrm{~g}$ of a $0.1 \mathrm{M}$ aqueous $\mathrm{AgNO}_{3}$ solution until the brown precipitate disappears. The thus prepared complex was thoroughly mixed with oleic acid and stirred for $1 \mathrm{~h}$ to obtain a defined molar ratio of oleic acid to $\mathrm{Ag}^{+}$. This led to the formation of a white, viscous gel of diamminesilver-(I) oleate (2).

For the reduction of the silver ions, $0.1 \mathrm{~g}$ of the complex 2 was dispersed in $5 \mathrm{~g}$ of ethanol to form an opalescent suspension. Subsequently, two drops of formaldehyde were added, followed by the slow and dropwise addition of $0.1 \mathrm{ml} 1 \mathrm{M} \mathrm{KOH}$ under continuous shaking at $300 \mathrm{rpm}$. The colour of the suspension changed from pale white to light gray, gray, and finally to dark gray with increasing volume of $\mathrm{KOH}$. After complete addition of the $\mathrm{KOH}$, the suspension was shaken for $1 \mathrm{~h}$.

\section{Results and discussion}

When mixing solutions of the diamminesilver-(I) complex with oleic acid (OA), different product morphologies are obtained depending on the molar ratio of $\mathrm{OA}$ to $\mathrm{Ag}^{+}$. Below $\mathrm{OA} / \mathrm{Ag}=$ 30 , the product agglomerates irreversibly and precipitates in water. Above $\mathrm{OA} / \mathrm{Ag}=30$, a homogenous, viscous white gel is obtained. Fig. 1 shows images of the dark-field optical microscopy for the diamminesilver-(I) oleate gel at an oleatesilver molar ratio of 30. At low magnification, the gel seems to consist of spherical aggregates [Fig. 1(a)]. Higher magnifications [Fig. 1(b)] reveal that these aggregates are composed of nanometre-sized strings, which agglomerate to form thicker bundles. When this gel is dispersed in ethanol, the agglomerated bundles separate into individual filaments [Fig. 1(c)].
In contrast to other objects composed of oleic acid moieties, these filaments are hard and break under the microscope when stressed.

Fig. 2 shows unstained TEM micrographs of the oleic acid$\mathrm{Ag}^{+}$mixtures. At a molar ratio of 30 (oleic acid : $\mathrm{Ag}^{+}$), large bundles of nanowires are formed [Fig. 2(a)]. These bundles are composed of solid cylindrical aggregates and hollow nanotubes as observed at higher magnification. The length ranges from 5 to $8 \mu \mathrm{m}$ and the diameters from 280 to $500 \mathrm{~nm}$. When the molar ratio of oleic acid to $\mathrm{Ag}^{+}$reaches 50, long nanowires with high aspect ratios are obtained [Fig. 2(b)]. High resolution TEM reveals that these nanowires possess hollow cavities [Fig. 2(c,d)]. The lengths of these nanotubes are in the order of several tens of microns, with outer diameters of 155 $200 \mathrm{~nm}$, i.e. an aspect ratio of $c a .250$. The wall thickness is 60-70 nm. Apparently, the molar ratio of OA : Ag directly
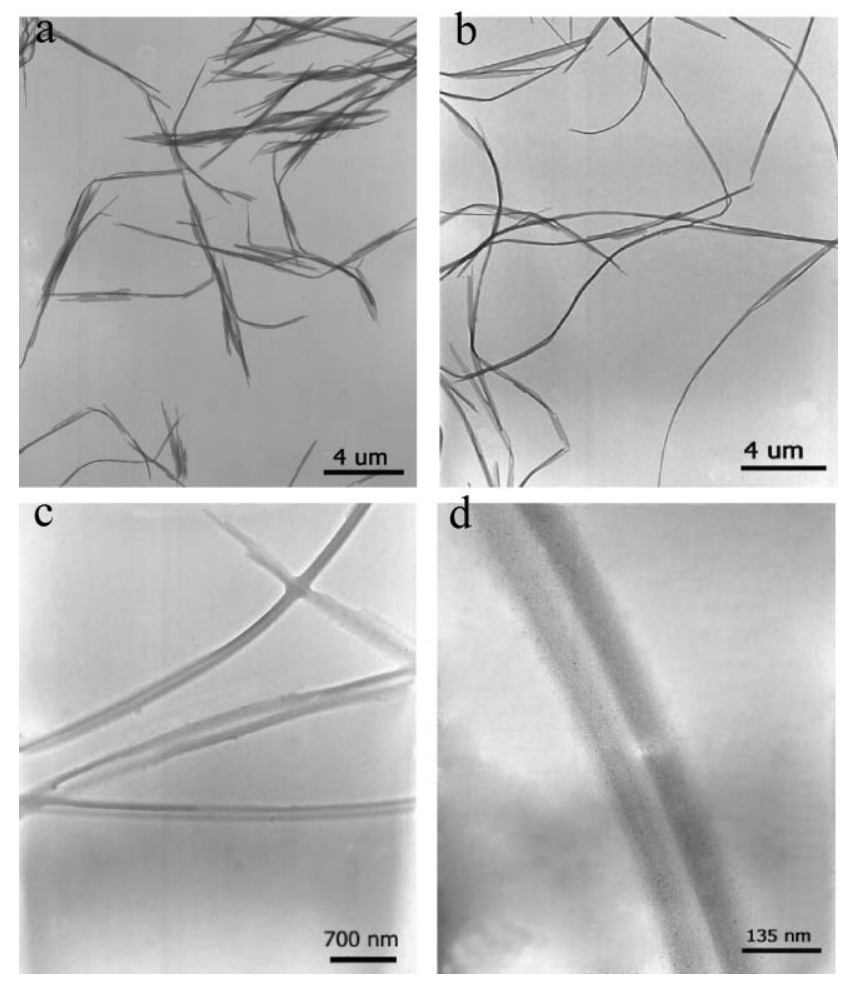

Fig. 2 TEM micrographs (unstained) of a diamminesilver-(I) oleate gel at a molar ratio of oleic acid to $\mathrm{Ag}^{+}$of 30 (a) and 50 (b-d). 
influences the dimensions of the tubular aggregates, which become thinner and longer with increasing OA content. The reduced cables seem to follow this trend. The ratio can, however, not be used to precisely control the cable's length or diameter, as the length and the diameter are subject to a certain distribution.

To form nanowires with a metallic core, the nanotube dispersions were treated with formaldehyde. As expected, the reaction conditions had a great influence on the morphology of the reduction products (Fig. 3). Apart from an oleic acid/ $\mathrm{Ag}^{+}$ratio of larger than 30 , gradual addition of the reagents under gentle agitation is required to obtain well-defined nanocables. The diameters of these cables match the ones found for the hollow tubes observed before reduction. In the TEM micrograph, a sharp contrast between sheath and core can be clearly observed. Since the structure-directing template, i.e. the self-assembled tube, becomes an integral part of the cable, it is obtained as an individually dispersed entity. The cores are straight and uniform with diameters of $c a$. 30-45 nm throughout their entire length. The silver profile across a nanocable [Fig. 3(d), bottom] further confirms the presence of a solid, inner silver core. However, the sheath does not seem to be entirely depleted of silver. The white spots in the sheath and the peaks in the profile indicate the presence of small silver particles. Additionally, unreacted $\mathrm{Ag}^{+}$-oleate in the sheath cannot be completely ruled out.

In contrast, if all the reagents are added at once under violent stirring, a different morphology is found (Fig. 4). The product now consists of short rods of $200 \mathrm{~nm}$ in length (aspect ratio $\sim 5$ ) and even spherical particles of $c a$. 10-70 $\mathrm{nm}$ in
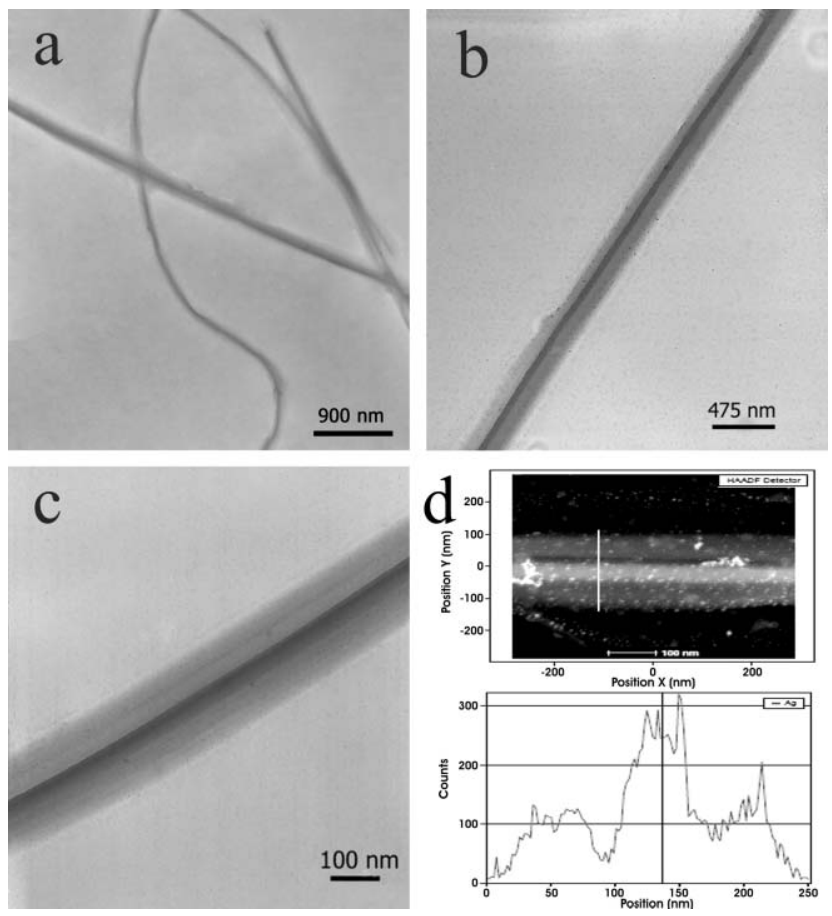

Fig. 3 Bright-field TEM micrographs of nanocables after reduction of a gel with a molar ratio of oleic acid to $\mathrm{Ag}^{+}$of $50(\mathrm{a}-\mathrm{c})$ and a darkfield TEM micrograph (d, top) and the corresponding silver profile across the nanocable (d, bottom).

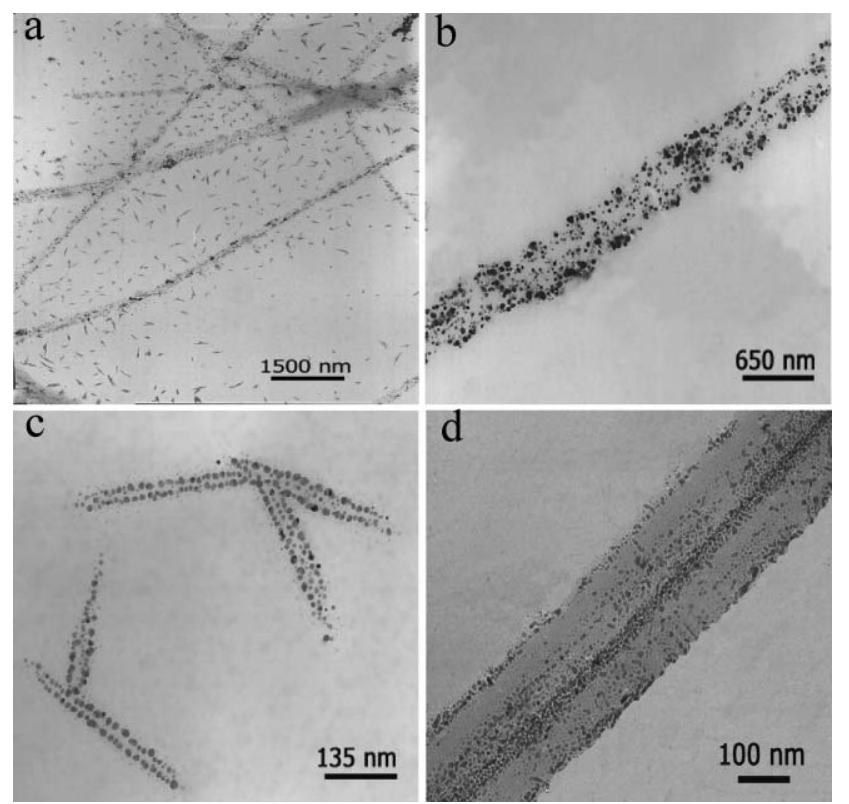

Fig. 4 TEM micrographs of the reduction products made by fast addition of $\mathrm{KOH}(\mathrm{a}-\mathrm{c})$ and substituting $\mathrm{NaBH}_{4}$ for formaldehyde (d).

diameter. Clearly, adding all the reducing agent at once induces fast nucleation, which results in the formation of spherical particles inside the oleate tubes. ${ }^{21}$ It is important to note that the spheres are not randomly distributed, but lined up bearing close resemblance to the original tubular structure [Fig. 4(b)]. Higher magnifications [Fig. 4(c)] reveal that the rod-like objects are also composed of small spherical nanoparticles of approximately $10 \mathrm{~nm}$ in diameter arranged like pearls on a string, while preserving the original tubular structure.

Similar results are obtained when formaldehyde is replaced by $\mathrm{NaBH}_{4}$. Instead of fully developed cables, again particles are found all over the tube [Fig. 4(d)]. Upon close examination, several features become evident: $(i)$ most particles are located in the core of the cable, (ii) the outer surface is densely covered with particles, and (iii) between the core and the surface, the particles are arranged in stripes parallel to the core. This situation is also seen when formaldehyde is used in low quantities, and appears to be an intermediate state on the way to forming fully developed cables. The question now arises as to how the tubes and cables are formed.

It has been reported that metal salts of long chain fatty acids form bilayer aggregates ${ }^{22}$ and can assemble into inverted cylindrical structures. ${ }^{23}$ The X-ray diffraction pattern of the nanotube suspensions shows one sharp reflection (100) at $3.18 \mathrm{~nm}$. An individual metal oleate is approximately $2 \mathrm{~nm}$ long, ${ }^{24}$ which suggests the presence of interdigitated bilayers. ${ }^{25}$ With an observed wall thickness of 60-70 nm, the sheath of the presented cables seems to be composed of approximately 20 layers. The layer theory is supported by the formation of particles in a string-like alignment within the sheath [Fig. 4(d)]. Theoretically, a tubular superstructure composed of a bundle of smaller tubes could also account for the observed string-like arrangement of particles, but such a structure was not seen in high resolution TEM. Furthermore, the inner core diameter 


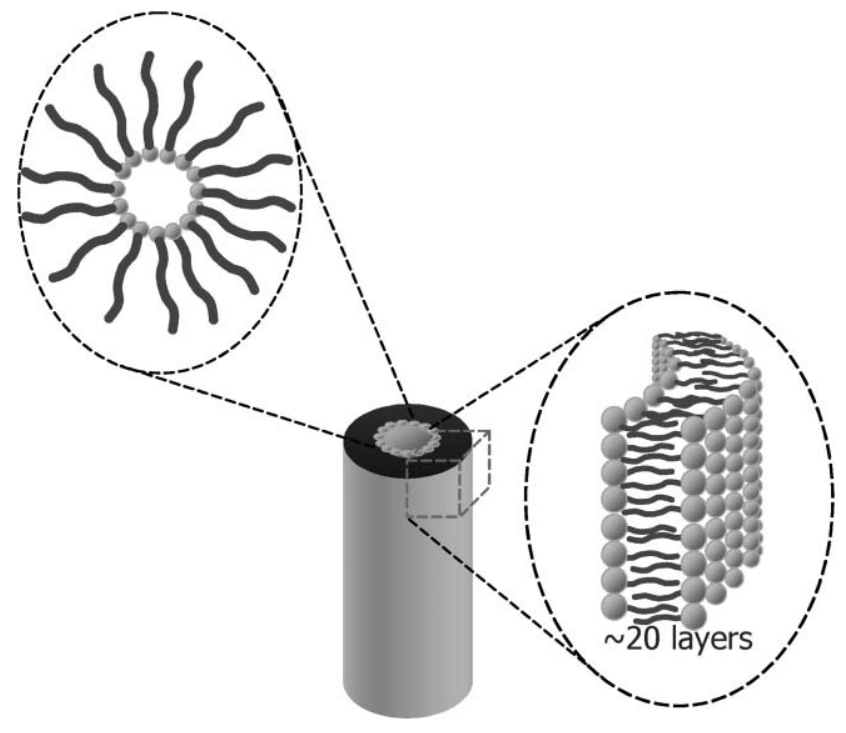

Fig. 5 Proposed schematic structure of the oleic acid $-\mathrm{Ag}^{+}$nanotubes.

seems to be the smallest arrangement of diamminesilver-(I) oleate with a sufficiently dense packing of the alkyl chains to form bilayers. The hollow core before reduction [Fig. 2(d)] and the silver wire [Fig. 3(c)] are, on the other hand, prominent features in the TEM micrographs, and a multi-bundle structure should, therefore, be clearly noticeable. Based on these considerations we assume the tubes to consist of a parallel multi lamellar wall structure as depicted in Fig. 5.

Upon addition of the reducing agent, the silver ions are reduced to metallic silver. As seen in Fig. 4(d), this nucleation might take place all over the tube. Slow addition of the reducing agent allows for Ostwald ripening and provides the mechanism for silver ions to be transported to the centre. As seen in Fig. 4(d), the particle density in the core is higher than anywhere else. This allows for the formation of large particles by agglomeration of smaller ones early in the process. These then grow at the expense of the remaining small particles in the structure. No particles outside the template were found in any of the experiments under the described conditions.

To gain more insight into the structure of the tubes, the FTIR spectra of the nanotubes were compared with that of oleic acid and the final nanocable (Fig. 6). The characteristic vibration of oleic acid is a broad $\mathrm{OH}$ stretching band at $\sim 3000 \mathrm{~cm}^{-1}$ and a sharp $\mathrm{C}=\mathrm{O}$ vibration at $1710 \mathrm{~cm}^{-1}$. These signals were not found in the spectra of the nanotubes. For the nanotube, the new strong signals at 1563,1467 , and $1419 \mathrm{~cm}^{-1}$ could be assigned to the asymmetric $\left(1563 \mathrm{~cm}^{-1}\right)$ and the symmetric (1467 and $1419 \mathrm{~cm}^{-1}$ ) stretching vibrations of the ionized carboxylate group. ${ }^{24}$ The characteristic signals of $\mathrm{NH}$ bending and stretching were also found at 1517 and $3410 \mathrm{~cm}^{-1}$, respectively.

The asymmetric and symmetric carboxylate vibrations can be used to determine the type of coordination by calculating the difference $\Delta=v_{\text {as }}(\mathrm{COO})-v_{\mathrm{s}}(\mathrm{COO}){ }^{25}$ The $\Delta$ values indicate the four main coordination types: ionic $\left(\Delta=164 \mathrm{~cm}^{-1}\right)$, monodentate $\left(\Delta=200-300 \mathrm{~cm}^{-1}\right)$, chelating bidentate $(\Delta=$ $\left.40-110 \mathrm{~cm}^{-1}\right)$, and bridging bidentate $\left(\Delta=140-170 \mathrm{~cm}^{-1}\right){ }^{25}$

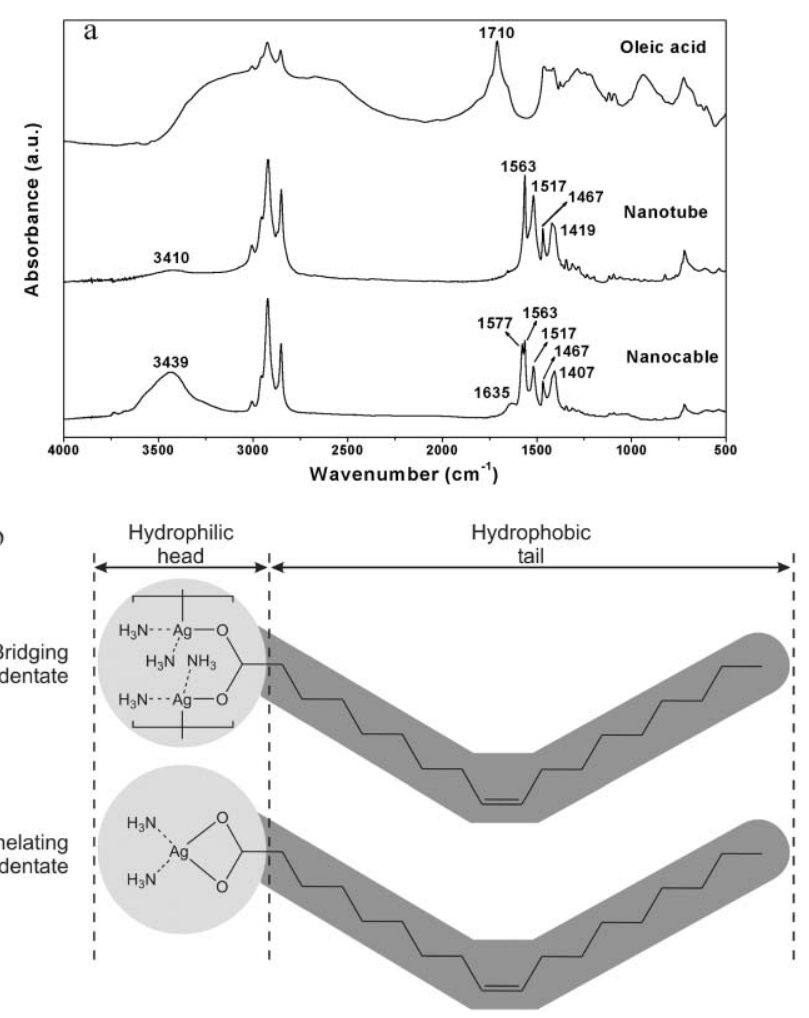

Fig. 6 FTIR spectra of oleic acid, the nanotubes, and silver nanocables (a) and the coordination types deduced from this (b).

In the case of the present nanotubes, there are two symmetric stretching vibrations, with the two $\Delta$ values of $\Delta_{1}=96$ and $\Delta_{2}=$ $144 \mathrm{~cm}^{-1}$. The first can be assigned to a chelating bidentate type, while the second one indicates a bridging bidentate [Fig. 6(b)]. These results further corroborate the formation of diamminesilver-(I) oleate. After reduction to nanocables, a slight change in the FTIR spectrum is observed compared to the nanotubes. A new signal at $1577 \mathrm{~cm}^{-1}$ appeared, while the signal at $1419 \mathrm{~cm}^{-1}$ shifted to $1407 \mathrm{~cm}^{-1}$, and the signal at $3410 \mathrm{~cm}^{-1}$ shifted to $3439 \mathrm{~cm}^{-1}$ and became stronger. In addition, a new peak at $1635 \mathrm{~cm}^{-1}$ was found, which might be assigned to the $\mathrm{C}=\mathrm{O}$ vibration of an amide group. It can, therefore, be safely assumed that the sheath consists of oleate molecules, which are partially cross-linked by silver atoms. This would explain their stiffness which is observed under the optical microscope.

\section{Conclusions}

In conclusion, we have demonstrated a convenient approach to preparing individual $\mathrm{Ag}$ nanocables using the self-assembly of tubular diamminesilver-(I) oleate as templates. To obtain the well-defined nanocables, two steps are important. First, in the preparation of the diamminesilver-(I) oleate precursors, the molar ratio of oleic acid/ $\mathrm{Ag}^{+}$must reach 50 , so that ordered nanotubes with high aspect ratios can be formed. Second, in order to avoid fast nucleation resulting in the formation of spherical silver particles, the reduction conditions must be carefully controlled by the slow and dropwise addition of the reducing agent under gentle shaking. The prepared nanotubes 
seem to consist of a multi-wall structure. During the reduction, silver particles seem to be formed initially and arranged in ribbon-like intermediate structures, finally leading to nanocables via Ostwald ripening and particle agglomeration. We believe that this approach could be extended to prepare a variety of metal nanocables by an appropriate choice of the inorganic/organic system and experimental conditions. In addition, metal nanowires also can be prepared by this method, since the organic sheath can be easily removed by dissolving in suitable organic solvents.

\section{Acknowledgements}

The authors thank D. A. Ivanov for conducting the X-ray scattering experiments. S.C.H. thanks the National Science Council of Taiwan and the DAAD (Deutscher Akademischer Austauschdienst) for a personal grant as part of the Sandwich programme. The authors gratefully acknowledge financial support from the Max-Buchner-Forschungsstiftung (research grant \#2470).

\section{References}

1 A. K. Salem, P. C. Searson and K. W. Leong, Nat. Mater., 2003, 2 , 668-671.

2 H. F. Wang, T. B. Huff, D. A. Zweifel, W. He, P. S. Low, A. Wei and J. X. Cheng, Proc. Natl. Acad. Sci. U. S. A., 2005, 102, $15752-15756$.

3 J. U. Lee, Appl. Phys. Lett., 2005, 87, 073101.

4 R. S. Friedman, M. C. McAlpine, D. S. Ricketts, D. Ham and C. M. Lieber, Nature, 2005, 434, 1085.

5 A. M. Morales and C. M. Lieber, Science, 1998, 279, 208-211.
6 Y. Zhang, K. Suenaga, C. Colliex and S. Iijima, Science, 1998, 281, 973-975.

7 W. S. Shi, H. Y. Peng, L. Xu, N. Wang, Y. H. Tang and S. T. Lee, Adv. Mater., 2000, 12, 1927-1928.

8 J. Q. Hu, X. Meng, M. Y. Jiang, C. S. Lee and S. T. Lee, $A d v$. Mater., 2003, 15, 70-71.

9 Y. D. Yin, Y. Lu, Y. G. Sun and Y. N. Xia, Nano Lett., 2002, 2, 427-430.

10 M. H. Huang, A. Choudrey and P. D. Yang, Chem. Commun., 2000, 1063-1064.

11 Y. Xie, Z. P. Qiao, M. Chen, X. M. Liu and Y. T. Qian, $A d v$. Mater., 1999, 11, 1512-1513.

12 L. B. Luo, S. H. Yu, H. S. Qian and T. Zhou, J. Am. Chem. Soc., 2005, 127, 2822-2823.

13 J. S. Jang, B. Lim, J. Lee and T. Hyeon, Chem. Commun., 2001, 83-84.

14 T. A. Crowley, B. Daly, M. A. Morris, D. Erts, O. Kazakova, J. J. Boland, B. Wu and J. D. Holmes, J. Mater. Chem., 2005, 15, 2408-2413.

15 M. Andersson, V. Alfredsson, P. Kjellin and A. E. C. Palmqvist, Nano Lett., 2002, 2, 1403-1407.

16 Q. T. Wang, G. Z. Wang, X. H. Han, X. P. Wang and J. G. Hou, J. Phys. Chem. B, 2005, 109, 23326-23329.

17 K. Takahashi, Y. Wang and G. Z. Cao, J. Phys. Chem. B, 2005, 109, 48-51.

18 S. H. Yu, S. H. Yu, X. J. Cui, L. L. Li, K. Li, B. Yu, M. Antonietti and H. Cölfen, Adv. Mater., 2004, 16, 1636-1637.

19 B. Yang, S. Kamiya, Y. Shimizu, N. Koshizaki and T. Shimizu, Chem. Mater., 2004, 16, 2826-2831.

20 D. Ugarte, A. Châtelain and W. A. de Heer, Science, 1996, 274, 1897-1899.

21 N. R. Jana, Small, 2005, 1, 875-882.

22 J. H. Fuhrhop, D. Spiroski and C. Boettcher, J. Am. Chem. Soc., 1993, 115, 1600-1601.

23 D. H. Gray and D. L. Gin, Chem. Mater., 1998, 10, 1827-1832.

24 H. Qiu and M. Caffrey, J. Phys. Chem. B, 1998, 102, 4819-4829.

25 G. John, M. Masuda, Y. Okada, K. Yase and T. Shimizu, $A d v$. Mater., 2001, 13, 715-718. 